\title{
In memoriam Ewald Weibel (1929-2019)
}

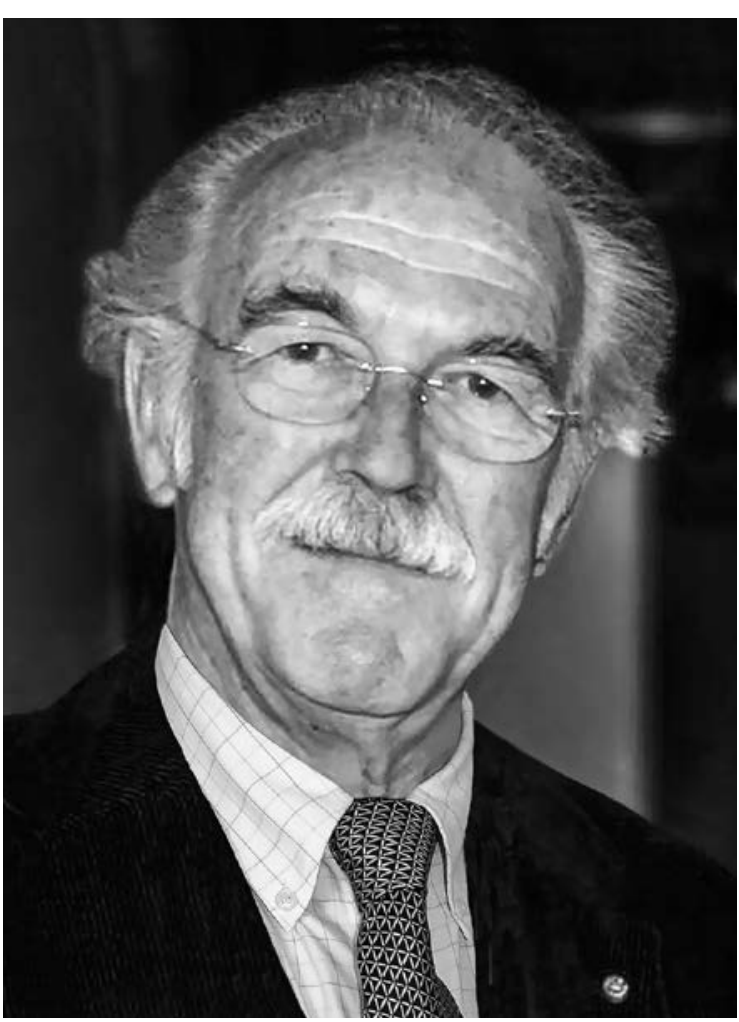

Ewald Weibel

Personnalité de premier plan du monde de la science et de la politique scientifique suisse et figure de proue de l'Académie Suisse des Sciences Médicales (ASSM), Ewald Weibel nous a quittés en début d'année.

L'homme et l'ami, l'enseignant académique, le mentor, le chercheur passionné et intègre resteront dans nos mémoires.

Un portrait très justement esquissé dans l'avis de décès. Ewald Weibel a incarné tout cela, mais bien plus encore pour l'ASSM qui lui dédie cet hommage. En tant que Président de 1997 à 2000, Vice-Président de 2000 à 2004, membre d'honneur et membre individuel du Sénat à partir de 2004, il a marqué l'Académie de son empreinte. En 1998, suite à la mort tragique et soudaine de Justus Gelzer, Secrétaire général en exercice, Ewald Weibel a entrepris une réorganisation profonde de l'ASSM. Il a professionnalisé le Secrétariat général qui fonctionnait jusqu'alors selon le principe du bénévolat et a accordé à la jeune équipe sa confiance totale pour amorcer une nouvelle direction. Cette nouvelle ère initiée par Ewald Weibel s'est également traduite de manière explicite dans l'identité visuelle de l'ASSM qui a été adaptée en 1999: le logo - un serpent qui symbolise la médecine, entouré d'une devise en latin - a été conservé, avec toutefois un graphisme modernisé et la devise "Scientiae Medicinali et Societati» a remplacé "Medici et Professoribus». Ainsi, sous la direction d'Ewald Weibel, l'ASSM a reconnu la nécessité d'élargir sa mission jusqu'alors uniquement dédiée à la profession médicale, à un engagement au service des patients et de la société.

Grâce à des projets innovants et porteurs, Ewald Weibel a consolidé et développé les fondements sur lesquels repose aujourd'hui encore l'ASSM: d'une part la promotion de la science médicale basée sur les preuves et, d'autre part, la pratique médicale et ses répercussions sur la société. Sous son égide, de nombreuses directives médico-éthiques vouées à des sujets brûlants à l'avènement du nouveau millénaire, tels que les xénotransplantations, la stérilisation de personnes handicapées ou la médecine intensive, ont vu le jour. C'est également à son initiative que l'ASSM a reconnu l'intégrité scientifique dans la recherche médicale et biomédicale comme un sujet prioritaire et défini, à l'intention des Facultés de médecine, une procédure à suivre

Sous son égide, de nombreuses directives médico-éthiques vouées à des sujets brûlants à l'avènement du nouveau millénaire.

en cas de fraudes. Avec son soutien, le projet «Nouvelle orientation de la médecine» a été lancé. Considérée comme une organisation scientifique intègre avec une sensibilité particulière pour les questions d'éthique médicale, l'ASSM a gagné en notoriété et a pu ainsi renforcer sa position au sein du corps médical et des autres professions de la santé, dans les milieux politiques et dans la société.

\section{De la science à la pratique}

Ewald Weibel était et reste un scientifique exceptionnel. Il a été distingué et honoré à maintes reprises dans le monde entier pour ses performances remarquables, entre autres par des titres de docteurs honoraires et des médailles d'universités renommées. Il a notamment obtenu le Prix Marcel Benoist en 1974 pour ses travaux pionniers dans le domaine de la morphologie fonctionnelle du poumon, et le titre de membre honoraire de l'American Academy of Arts and Sciences en 
2000. Ses nombreuses interventions dans des congrès à orientation clinique, dont l'«American Thoracic Society» et la "European Respiratory Society" pendant des années, témoignent de l'importance de ses travaux de recherche et de ses connaissances pour la pratique. Ses travaux en faveur de la compréhension de la morphologie fonctionnelle du poumon lui ont valu de nombreux hommages. Ewald Weibel était un pionnier dans son domaine de recherche, les relations entre la structure et la fonction du poumon et finalement de tout l'organisme. De telles études des relations n'ont toutefois été possibles que grâce à la quantification des structures, la morphométrie. Pour ce faire, Ewald Weibel a développé une méthode mathématique et statistique, la stéréologie, qui est actuellement utilisée dans le monde entier dans la recherche sur les relations entre structure et fonction dans tous les tissus et organes. Ce n'est qu'ainsi qu'il a été possible de comparer les structures microscopiques de lumière et d'électrons enregistrées quantitativement avec les fonctions mesurées.

Ewald Weibel a également été un pionnier de la microscopie de lumière et particulièrement d'électrons. C'est grâce à lui que l'Institut d'anatomie est aujourd'hui le

\section{Ewald Weibel s'est beaucoup investi afin de créer} la possibilité de transposer les connaissances fondamentales dans l'application clinique.

Centre de microscopie de l'Université de Berne. Il convient également de mentionner les livres qu'il a écrits sur ce sujet, tels que Morphometry of the Human Lung (1963), The Pathway for Oxygen (1984) et Symmorphosis: On Form and Function in Shaping Life (2000). En travaillant étroitement, pendant de nombreuses années, avec des cliniciens, Ewald Weibel s'est beaucoup investi afin de créer la possibilité de transposer les connaissances fondamentales dans l'application clinique. Les praticiens ont eux aussi suivis avec intérêt ses découvertes sur les relations entre structure et fonction. Il avait le don extraordinaire de transmettre ses conclusions scientifiques d'une manière simple et claire. Enfin et surtout, pendant des décennies, un nombre impressionnant d'étudiants ont assisté avec enthousiasme à ses cours magistraux. Ewald Weibel était non seulement un scientifique exceptionnel, mais aussi un enseignant talentueux.

Celles et ceux qui ont eu le privilège de travailler avec Ewald Weibel ont également connu et apprécié

\section{Il avait le don extraordinaire de transmettre} ses conclusions scientifiques d'une manière simple et claire.

l'homme. Son énergie inépuisable, son autorité naturelle, son enthousiasme pour sa propre discipline comme pour toutes les questions scientifiques et médicales, son sens de l'essentiel, son esprit affûté, mais également son grand cœur, son charme, son empathie et son sens de l'humour étaient impressionnants.

Une personnalité telle qu'Ewald Weibel suscite un respect profond et la reconnaissance de toutes celles et de tous ceux qui ont eu le privilège de faire un bout de chemin avec lui.

Merci, Ewald!

$$
\begin{array}{r}
\text { Margrit Leuthold, } \\
\text { Secrétaire générale de l'ASSM 1998-2006 } \\
\text { Werner Stauffacher, } \\
\text { Président de l'ASSM 2000-2004 } \\
\text { Peter Gehr, } \\
\text { Professeur émérite, } \\
\text { Co-Directeur de l'Institut d'anatomie } \\
\text { de l'Université de Berne 2003-2010 }
\end{array}
$$

\section{Crédit photo}

https://de.wikipedia.org/wiki/Ewald Weibel\#/media/Datei:ERW portrait_2009.jpg. Diese Datei ist unter der «Creative Commons Attribution 3.0 Unported» lizenziert. Das Bild war ursprünglich farbig und wurde von der Medienmacher AG in Graustufen geändert. 